\title{
Billiard and diophantine approximation
}

\author{
by \\ JAN FLOREK (Wrocław)
}

1. Introduction. For a real number $x,[x]$ is the integral part of $x,\{x\}$ is the fractional part of $x$ and $\|x\|=\min (\{x\}, 1-\{x\})$ is the distance of $x$ to the nearest integer.

Let $0<\theta<1$ and $0 \leq \alpha<1$. A fraction $p / q, q>0$, is called a best $\alpha$-approximation to $\theta$ (homogeneous for $\alpha=0$ ) (see [4]) if

$$
\|q \theta-\alpha\|=|q \theta-\alpha-p|,
$$

and if

$$
\|j \theta-\alpha\|>\|q \theta-\alpha\| \quad \text { for } 0<j<q .
$$

Notice that if $\|q \theta-\alpha\|=0$ for some $q \in \mathbb{N}$, then the set of all best $\alpha$ approximations to $\theta$ is finite. It is well known [4] that the best homogeneous approximations to $\theta$ are given by the continued fraction process. Namely, the convergents $p_{n} / q_{n}$ to $\theta$ are the best homogeneous approximations to $\theta$, for $n \geq 1$ if $0<\theta \leq 1 / 2$ and for $n \geq 2$ if $1 / 2<\theta<1$ (see Remark 3.3). The idea of a best inhomogeneous approximation $(\alpha>0)$ has been investigated by several authors, for example Khintchine [7], Barnes and Swinnerton-Dyer [2], Cassels [3], Sós [11], Cusick, Rockett and Szüsz [5] or Komatsu [8].

We say that an index $q$ is a critical index of a real-valued sequence $G(j)$, $j \in \mathbb{N}$, if

$$
G(j)>G(q) \quad \text { for } 0<j<q .
$$

We say that sequences $G(j)$ and $H(j), j \in \mathbb{N}$, are diophantine equivalent if they have the same set of critical indices and are equal on this set. Note that a fraction $p / q$ is a best $\alpha$-approximation to $\theta$ iff $q$ is a critical index of the sequence $\|j \theta-\alpha\|, j \in \mathbb{N}$. Hence, the sequence of the best $\alpha$-approximations to $\theta$ is determined by any sequence diophantine equivalent to the sequence $\|j \theta-\alpha\|, j \in \mathbb{N}$.

2000 Mathematics Subject Classification: 11A55, 11J20, 37A99.

Key words and phrases: inhomogeneous diophantine approximation, five distance theorem. 
A $(\theta, \alpha)$-billiard sequence (homogeneous for $\alpha=0$ ) is a sequence $F(j) \in$ $[0,1), j \in \mathbb{N}$, which satisfies the following conditions:

$$
\begin{array}{ll}
F(1)=\alpha / 2, & \\
F(j)+F(j+1)=\theta \text { or } 1+\theta & \text { for } j \text { odd, } \\
F(j)+F(j+1)=1 \text { or } 0 & \text { for } j \text { even. }
\end{array}
$$

Note that if $\alpha$ and $\theta$ are rational, then $F(j)$ is a periodic sequence. We consider a billiard table rectangle with the bottom left vertex labelled $v_{0}$, and the others, in a clockwise direction, $v_{1}, v_{2}$ and $v_{3}$. The distance from $v_{0}$ to $v_{1}$ is $\theta / 2$. We describe the position of points on the perimeter by their distance around the perimeter measured in a clockwise direction from $v_{0}$, so that $v_{1}$ is at position $\theta / 2, v_{2}$ at $1 / 2$ and $v_{3}$ at $(\theta+1) / 2$. If a billiard ball is sent out from position $F(1)=\alpha / 2$ at an angle of $\pi / 4$, then the ball will rebound against the sides of the rectangle consecutively at points $F(2), F(3), \ldots$.

Let $F(j), j \in \mathbb{N}$, be a $(\theta, \alpha)$-billiard sequence. We define the following sequences:

$$
\begin{aligned}
& A(j)= \begin{cases}\min \left\{\left\|F(j)-v_{i}\right\|: 0 \leq i \leq 3\right\} & \text { for } j>1, \\
\min \left\{\left\|F(j)-v_{i}\right\|: i=1,3\right\} & \text { for } j=1,\end{cases} \\
& B(j)=\min \left\{\left\|F(j)-v_{i}\right\|: 1 \leq i \leq 3\right\}, \quad j \in \mathbb{N}, \\
& C(j)=\min \{\|F(k)-F(l)\|: 1 \leq k<l \leq j+1\}, \quad j \in \mathbb{N} .
\end{aligned}
$$

Since $\|x-y\|=\min (\{x-y\}, 1-\{x-y\})$ is the shortest perimeter distance between $x, y \in[0,1)$, the value $A(j), j>1$, is the distance between the rebound $F(j)$ and the set of vertices of the rectangle, and $C(j)$ is the minimal distance between any two rebounds $F(k)$ and $F(l)$ for $1 \leq k<l \leq j+1$.

In Theorem 3.1(1) we prove that $\|j \theta-\alpha\|=\|F(j)-F(j+1)\|$ for $j \in \mathbb{N}$. The main aim of this paper is to prove Theorem 3.2: the sequences $\|j \theta-\alpha\|, 2 A(j)$ are diophantine equivalent, and so too are the sequences $C(j)$, $\min (2 A(j),\|F(j+1)-F(1)\|)$. In the homogeneous case all the above sequences are diophantine equivalent to the sequence $2 B(j), j \in \mathbb{N}$.

In Theorem 3.3 we prove that if $p / q, q>1$, is a best $\alpha$-approximation to $\theta$, then the numbers $(-1)^{p},(-1)^{q}$ determine the unique vertex $v(q)$ such that $\|q \theta-\alpha\|=2\|F(q)-v(q)\|$. In Corollaries 3.2 and 3.3 we consider the homogeneous case: if $p_{n} / q_{n}$ and $a_{n}, n \in \mathbb{N}$, are the sequences of convergents and partial quotients to $\theta<1 / 2$, then the sequences $v\left(q_{n}\right)$ and $\operatorname{sgn}\left(F\left(q_{n}\right)-v\left(q_{n}\right)\right)$ are determined by the sequence $(-1)^{a_{n}}, n \in \mathbb{N}$. On the other hand,

$$
a_{n}=\left[\frac{\left\|q_{n-1} \theta\right\|}{\left\|q_{n} \theta\right\|}\right]=\left[\frac{\left\|F\left(q_{n-1}\right)-v\left(q_{n-1}\right)\right\|}{\left\|F\left(q_{n}\right)-v\left(q_{n}\right)\right\|}\right] \quad \text { for } n>1 .
$$

The following theorem is known as the Steinhaus conjecture or the three 
distance theorem: there are at most three lengths when the unit circle is partitioned by the points $\{j \theta\}$ for $1 \leq j \leq n$. This theorem was first proved by Sós [11], [12] and then by Świerczkowski [14] and Surányi [13] (see also [1]). Surányi formulates this result in terms of $n$-Farey points. Ravenstein [10] gives solutions in terms of "best" and "second best" rational approximations to $\theta$. Geelen and Simpson [6] prove the following five distance theorem: there are at most five lengths when the unit circle is partitioned by the points $\{j \theta\}$ and $\{j \theta+\beta\}$ for $0 \leq j \leq n$.

In Theorem 2.1 we give an explicit formula for a $(\theta, \alpha)$-billiard sequence. It follows that the five (three) distance theorem is equivalent to the following: there are at most five lengths (three if $F(1)=0$ ) when the perimeter of the rectangle is partitioned by a finite sequence of successive rebounds of a billiard ball.

2. Billiard and the five distance theorem. For real numbers $x, y$ we write

$$
x \equiv y \quad \text { iff } \quad x-y \text { is an integral number. }
$$

Theorem 2.1. Let $0<\theta<1$ and $0 \leq \alpha<1$. A sequence $F(j) \in[0,1)$, $j \in \mathbb{N}$, is a $(\theta, \alpha)$-billiard sequence iff it satisfies the following conditions:

$$
\begin{array}{ll}
F(2 n)=\{n \theta-\alpha / 2\} & \text { for } n \in \mathbb{N}, \\
F(2 n+1)=\{-n \theta+\alpha / 2\} & \text { for } n \in \mathbb{N} \cup\{0\} .
\end{array}
$$

Proof. If the above equalities are satisfied, then

$$
\begin{aligned}
& F(2 n-1)+F(2 n) \equiv-(n-1) \theta+\alpha / 2+n \theta-\alpha / 2=\theta, \\
& F(2 n)+F(2 n+1) \equiv n \theta-\alpha / 2-n \theta+\alpha / 2=0 .
\end{aligned}
$$

Since $0 \leq F(2 n-1)+F(2 n)<2$, we get $F(2 n-1)+F(2 n)=\theta$ or $1+\theta$. Since $0 \leq F(2 n)+F(2 n+1)<2$, we get $F(2 n)+F(2 n+1)=0$ or 1 .

Conversely, we prove, by induction, that the $(\theta, \alpha)$-billiard sequence satisfies the condition of Theorem 2.1:

$$
\begin{aligned}
& F(2 n) \equiv-F(2 n-1)+\theta \equiv(n-1) \theta-\alpha / 2+\theta=n \theta-\alpha / 2, \\
& F(2 n+1) \equiv-F(2 n) \equiv-n \theta+\alpha / 2 .
\end{aligned}
$$

This theorem shows that the set of values of a $(\theta, \alpha)$-billiard sequence is the union of two sets of points placed consecutively around the circle an angle $\theta$ apart in two opposite directions. Hence the five (three) distance theorem is equivalent to the following corollary:

COROLlaRY 2.1. There are at most five lengths (three if $F(1)=0$ ) when the perimeter of the rectangle is partitioned by a finite sequence of successive rebounds of a billiard ball. Here "length" means the distance around the perimeter between adjacent rebound points. 
For a family $\mathcal{L}$ of sets, a member $S \in \mathcal{L}$ is defined to be a minimal set if it does not contain any other member of $\mathcal{L}$.

COROLlary 2.2. The trajectory obtained by a finite sequence of successive rebounds of a billiard ball "draws" at most 15 incongruent and minimal rectangles: 5 squares and $\left(\begin{array}{l}5 \\ 2\end{array}\right)$ non-square rectangles (at most $3+\left(\begin{array}{l}3 \\ 2\end{array}\right)=6$ if $F(1)=0)$.

REMARK 2.1. Consider a general case, when the initial angle of the ball's motion is not $\pi / 4$. By a linear transformation $L$ (compressing or stretching) we can change the billiard table rectangle, so that the general case is transformed to the $\pi / 4$ case of Corollary 2.2. Any square which appears in Corollary 2.2 is transformed by $L^{-1}$ into a rhombus. By analogy, any pair of rectangles with the perpendicular sides of the same length is transformed into a pair of parallelograms which are mirror images of each other. Thus we get at most 25 incongruent and minimal parallelograms: 5 rhombi and 10 pairs of parallelograms which are mirror images of each other. There are also at most 5 incongruent triangles adjacent to the perimeter in the $\pi / 4$ case and 10 in the general case.

3. Billiard and the best approximations. Let $0<\theta<1,0 \leq \alpha<1$, let $F(j)$ be the $(\theta, \alpha)$-billiard sequence, and let $A(j), B(j), C(j), j \in \mathbb{N}$, be the sequences defined in the Introduction.

Lemma 3.1. For a real number $x$,

$$
\|x\|=\min \left(2\left\|\frac{1}{2} x\right\|, 2\left\|\frac{1}{2}(x-1)\right\|\right) .
$$

Proof. If $2 n<x<2 n+1, n \in \mathbb{Z}$, then

$$
\{x\}=x-2 n=2\left\|\frac{1}{2} x\right\| \quad \text { and } \quad\{-x\}=2 n-(x-1)=2\left\|\frac{1}{2}(x-1)\right\| .
$$

If $2 n-1<x<2 n, n \in \mathbb{Z}$, then

$$
\{x\}=x+1-2 n=2\left\|\frac{1}{2}(x+1)\right\| \quad \text { and } \quad\{-x\}=2 n-x=2\left\|\frac{1}{2} x\right\| .
$$

Hence

$$
\|x\|=\min (\{x\},\{-x\})=\min \left(2\left\|\frac{1}{2} x\right\|, 2\left\|\frac{1}{2}(x-1)\right\|\right) .
$$

Notice that $\|x\|=\|y\|$ iff $x \equiv y$ or $x \equiv-y$.

Theorem 3.1.

(1) $\|j \theta-\alpha\|=\|F(j)-F(j+1)\|$.

(2) $\|j \theta-\alpha\|= \begin{cases}\min \left(2\left\|F(j)-\frac{1}{2} \theta\right\|, 2\left\|F(j)-\frac{1}{2}(1+\theta)\right\|\right) & \text { for } j \text { odd, } \\ \min \left(2\|F(j)\|, 2\left\|F(j)-\frac{1}{2}\right\|\right) & \text { for } j \text { even. }\end{cases}$

Proof. By Theorem 2.1 we have the following equalities: $F(2 n+1)-F(2 n+2) \equiv-n \theta+\alpha / 2-[(n+1) \theta-\alpha / 2]=-(2 n+1) \theta+\alpha$, 


$$
F(2 n)-F(2 n+1) \equiv n \theta-\alpha / 2-(-n \theta+\alpha / 2)=2 n \theta-\alpha .
$$

Hence (1) follows.

Theorem 2.1 also yields the following equalities:

$$
2 F(2 n+1)-\theta \equiv-(2 n+1) \theta+\alpha, \quad 2 F(2 n) \equiv 2 n \theta-\alpha .
$$

Hence by Lemma 3.1 we obtain (2).

Lemma 3.2. Sequences $G(j)$ and $H(j), j \in \mathbb{N}$, are diophantine equivalent iff for every $j \in \mathbb{N}$ there exist $j_{1}, j_{2} \leq j$ such that

$$
G\left(j_{1}\right) \leq H(j) \quad \text { and } \quad H\left(j_{2}\right) \leq G(j) .
$$

Proof. Assume that $G(j)$ and $H(j), j \in \mathbb{N}$, are diophantine equivalent. If $j$ is not a critical index, then there exists a critical index $q<j$ such that $H(q)=G(q) \leq \min \{H(j), G(j)\}$. If $j$ is a critical index then both inequalities are satisfied with $j_{1}=j_{2}=j$.

Now we prove the converse. Let $q$ be a critical index for the sequence $G(j)$. Since $G\left(j_{1}\right) \leq H\left(j_{2}\right) \leq G(q)$ for some $1 \leq j_{1} \leq j_{2} \leq q$, we have $j_{1}=j_{2}=q$ and $H(q)=G(q)$. If $q$ is not a critical index for the sequence $H(j)$, then we obtain the contradiction $G\left(i_{1}\right) \leq H\left(i_{2}\right) \leq H(q)=G(q)$ for some $1 \leq i_{1} \leq$ $i_{2}<q$. By analogy, if $q$ is a critical index for $H(j)$, then $H(q)=G(q)$ and $q$ is a critical index for $G(j)$.

By the definition of a $(\theta, \alpha)$-billiard sequence we obtain the following:

REMARK 3.1. For $j$ even,

$$
F(j)-\frac{1}{2} \theta \equiv \frac{1}{2} \theta-F(j-1) \quad \text { and } \quad F(j)-\frac{1}{2}(\theta+1) \equiv \frac{1}{2}(\theta+1)-F(j-1) .
$$

For $j>1$ odd,

$$
F(j) \equiv-F(j-1) \quad \text { and } \quad F(j)-\frac{1}{2} \equiv \frac{1}{2}-F(j-1) .
$$

REMARK 3.2.

$$
F(k)-F(l) \equiv \begin{cases}F(l-1)-F(k+1) & \text { for } 1 \leq k<l \text { of different parity } \\ F(l+1)-F(k+1) & \text { for } 1 \leq k<l \text { of the same parity }\end{cases}
$$

LEMMA 3.3. Let $j \in \mathbb{N}$.

(1) There exists $1 \leq j_{1} \leq j$ such that

$$
C(j)=\min \left(\left\|F\left(j_{1}+1\right)-F\left(j_{1}\right)\right\|,\left\|F\left(j_{1}+1\right)-F(1)\right\|\right) .
$$

(2) If $F(1)=0$, then there exists $1 \leq j_{2} \leq j$ such that

$$
C(j)=\left\|F\left(j_{2}+1\right)-F\left(j_{2}\right)\right\| \quad \text { and } \quad\left\|F\left(j_{2}\right)\right\| \neq 0 \quad \text { for } j_{2}>1 \text {. }
$$

Proof. By Remark 3.2 we have

$$
\|F(k)-F(l)\|= \begin{cases}\left\|F\left(k+\frac{l-k+1}{2}\right)-F\left(l-\frac{l-k+1}{2}\right)\right\| & \text { for } 0<l-k \text { odd } \\ \|F(1)-F(l-k+1)\| & \text { for } 0<l-k \text { even. }\end{cases}
$$

Hence (1) follows. 
Let $F(1)=0$. If $0<l-k$ is even, then by Remarks 3.2 and 3.1,

$$
\begin{aligned}
\|F(k)-F(l)\| & =\|F(1)-F(l-k+1)\|=\|F(1)-F(l-k)\| \\
& =\left\|F\left(1+\frac{l-k}{2}\right)-F\left(\frac{l-k}{2}\right)\right\| .
\end{aligned}
$$

Hence there exists the minimal number $1 \leq j_{2} \leq j$ such that $\| F\left(j_{2}+1\right)$ $-F\left(j_{2}\right) \|=C(j)$. If $j_{2}>1$ and $F\left(j_{2}\right)=0$, then by Remark 3.1 there exists an even $i \leq j_{2}$ such that $F(i)=0$. Thus by Remark 3.2 we obtain a contradiction $0=\|F(i)-F(1)\|=\left\|F\left(\frac{1}{2} i+1\right)-F\left(\frac{1}{2} i\right)\right\|>C(j)$. This yields (2).

THEOREM 3.2 .

(1) The sequences $\|j \theta-\alpha\|$ and $2 A(j), j \in \mathbb{N}$, are diophantine equivalent.

(2) The sequences $C(j)$ and $\min (2 A(j),\|F(j+1)-F(1)\|), j \in \mathbb{N}$, are diophantine equivalent.

(3) If $F(1)=0$, then the sequences $\|j \theta\|, C(j)$ and $2 B(j), j \in \mathbb{N}$, are diophantine equivalent.

Proof. By Remark 3.1 we have:

$$
\begin{aligned}
\min \left(2\left\|F(j)-\frac{1}{2} \theta\right\|, 2\left\|F(j)-\frac{1}{2}(1+\theta)\right\|\right) & \\
& =\min \left(2\left\|F(j-1)-\frac{1}{2} \theta\right\|, 2\left\|F(j-1)-\frac{1}{2}(1+\theta)\right\|\right)
\end{aligned}
$$

for $j$ even, and

$$
\min \left(2\|F(j)\|, 2\left\|F(j)-\frac{1}{2}\right\|\right)=\min \left(2\|F(j-1)\|, 2\left\|F(j-1)-\frac{1}{2}\right\|\right)
$$

for $j>1$ odd. Hence by Theorem 3.1(2) we have

$$
\left\{\begin{array}{l}
2 A(j)=\min (\|(j-1) \theta-\alpha\|,\|j \theta-\alpha\|) \quad \text { for } j>1, \\
2 A(1)=\|\theta-\alpha\| .
\end{array}\right.
$$

Thus by Lemma 3.2 condition (1) holds.

By Theorem 3.1(1) and (i) we have

$$
C(j) \leq \min (2 A(j),\|F(j+1)-F(1)\|) \quad \text { for } j \in \mathbb{N} .
$$

By Lemma 3.3(1) and Theorem 3.1, for every $j \in \mathbb{N}$ there exists $1 \leq j_{1} \leq j$ such that

$$
\begin{aligned}
C(j) & =\min \left(\left\|F\left(j_{1}+1\right)-F\left(j_{1}\right)\right\|,\left\|F\left(j_{1}+1\right)-F(1)\right\|\right) \\
& = \begin{cases}\min \left(2\left\|F\left(j_{1}\right)-\frac{1}{2} \theta\right\|, 2\left\|F\left(j_{1}\right)-\frac{1}{2}(1+\theta)\right\|,\left\|F\left(j_{1}+1\right)-F(1)\right\|\right) \\
\min \left(2\left\|F\left(j_{1}\right)\right\|, 2\left\|F\left(j_{1}\right)-\frac{1}{2}\right\|,\left\|F\left(j_{1}+1\right)-F(1)\right\|\right) & \text { for } j_{1} \text { odd },\end{cases} \\
& \geq \min \left(2 A\left(j_{1}\right),\left\|F\left(j_{1}+1\right)-F(1)\right\|\right) .
\end{aligned}
$$

Hence by Lemma 3.2 and (ii) condition (2) is satisfied.

Let $F(1)=0$. By (ii) we have

$$
C(j) \leq 2 A(j) \leq 2 B(j) \text { for } j \in \mathbb{N} .
$$


By Lemma 3.3(2) and Theorem 3.1, for every $j \in \mathbb{N}$ there exists $1 \leq j_{2} \leq j$ such that

$$
\begin{aligned}
C(j) & =\left\|F\left(j_{2}+1\right)-F\left(j_{2}\right)\right\| \\
& = \begin{cases}\min \left(2\left\|F\left(j_{2}\right)-\frac{1}{2} \theta\right\|, 2\left\|F\left(j_{2}\right)-\frac{1}{2}(1+\theta)\right\|\right) & \text { for } j_{2} \text { odd }, \\
\min \left(2\left\|F\left(j_{2}\right)\right\|, 2\left\|F\left(j_{2}\right)-\frac{1}{2}\right\|\right) & \text { for } j_{2} \text { even. }\end{cases}
\end{aligned}
$$

Since $C(j) \leq\left\|F\left(j_{2}\right)\right\| \neq 0$ for $j_{2}>1$, we have

$$
C(j) \geq 2 B\left(j_{2}\right) \text {. }
$$

Hence, by Lemma 3.2, (iii) and (1), condition (3) holds.

By Theorem 3.2(3) we obtain the following Dirichlet approximation theorem [4].

Corollary 3.1. Let $\theta$ and $\Theta>1$ be real. Then there is an integer $q$ such that

$$
0<q<\Theta, \quad\|q \theta\| \leq \Theta^{-1} .
$$

Proof. If $F(1)=0$ and $q_{n}, n \in \mathbb{N}$, is the increasing sequence of all critical indices of the sequence $C(j), j \in \mathbb{N}$, then by Theorem 3.2(3), $\left\|q_{n} \theta\right\|=C\left(q_{n}\right)$. Consider $n$ such that $q_{n}<\Theta \leq q_{n+1}$. Since $C(j) \leq(j+1)^{-1}$ for $j \in \mathbb{N}$, we have

$$
\left\|q_{n} \theta\right\|=C\left(q_{n}\right) \leq C\left(q_{n+1}-1\right) \leq q_{n+1}^{-1} \leq \Theta^{-1} .
$$

REMARK 3.3 (see [4]). Let integers $p_{n}, q_{n}, a_{n}$ be defined by

$$
\left\{\begin{array} { l } 
{ p _ { 0 } = 1 , q _ { 0 } = 0 , } \\
{ p _ { 1 } = 0 , q _ { 1 } = 1 , }
\end{array} \quad \left\{\begin{array}{l}
p_{n+1}=a_{n} p_{n}+p_{n-1}, \\
q_{n+1}=a_{n} q_{n}+q_{n-1}
\end{array} \text { for } n \geq 1,\right.\right.
$$

where

$$
a_{n}=\left[\frac{\left|q_{n-1} \theta-p_{n-1}\right|}{\left|q_{n} \theta-p_{n}\right|}\right]
$$

if $q_{n} \theta \neq p_{n}$, and the process stops with $p_{n}, q_{n}$ if $q_{n} \theta=p_{n}$. Then the $p_{n} / q_{n}$ are the best homogeneous approximations to $\theta$ for $n \geq 1$ if $0<\theta \leq 1 / 2$ and for $n \geq 2$ if $1 / 2<\theta<1$. Further,

$$
\begin{gathered}
(-1)^{n}\left(q_{n} \theta-p_{n}\right) \geq 0, \\
q_{n+1} p_{n}-q_{n} p_{n+1}=(-1)^{n} .
\end{gathered}
$$

It is usual to speak of the $p_{n} / q_{n}$ as the $n$th convergents to $\theta$ and to call the $a_{n}$ the partial quotients. Since the $a_{n}$ are determined by $\theta$ and $\theta=\lim p_{n} / q_{n}$, we may write $\theta=\left[0 ; a_{1}, a_{2}, \ldots\right]$.

REMARK 3.4. Let $0<\theta<1 / 2$. Homogeneous $\theta$ and $(1-\theta)$-billiard sequences have symmetrical interpretation in the billiard rectangle with sides of length $\frac{1}{2} \theta$ and $\frac{1}{2}(1-\theta)$. Hence by Theorem 3.2(3) the sequences $\|j \theta\|$ and $\|j(1-\theta)\|, j \in \mathbb{N}$, have the same set of critical indices. One can confirm this 
in terms of convergents to $\theta$ and to $1-\theta$. Let $p_{n} / q_{n}$ and $\bar{p}_{n} / \bar{q}_{n}, n \in \mathbb{N}$, be the $n$th convergents to $\theta$ and $1-\theta$ respectively. If $\theta=\left[0 ; a_{1}, a_{2}, \ldots\right], a_{1}>1$, then by the Lagrange formula [9], $1-\theta=\left[0 ; \bar{a}_{1}, \bar{a}_{2}, \ldots\right]=\left[0 ; 1, a_{1}-1\right.$, $\left.a_{2}, a_{3}, \ldots\right]$. Hence, by $\mathrm{CF}(1)$ and by induction we obtain

$$
\begin{aligned}
& \bar{q}_{2}=\bar{a}_{1} \bar{q}_{1}+\bar{q}_{0}=1=q_{1}, \\
& \bar{q}_{3}=\bar{a}_{2} \bar{q}_{2}+\bar{q}_{1}=\left(a_{1}-1\right) q_{1}+1=a_{1}=q_{2} \text {, } \\
& \bar{q}_{n+1}=\bar{a}_{n} \bar{q}_{n}+\bar{q}_{n-1}=a_{n-1} q_{n-1}+q_{n-2}=q_{n} \text { for } n \geq 3 .
\end{aligned}
$$

Lemma 3.4. If $q \theta-\alpha=p+2 d$, where $q \in \mathbb{N}, p \in \mathbb{N} \cup\{0\}$, then

$$
F(q) \equiv \begin{cases}d & \text { for } p \text { and } q \text { both even, } \\ \theta / 2-d & \text { for } p \text { even and } q \text { odd, } \\ 1 / 2+d & \text { for } p \text { odd and } q \text { even } \\ \frac{1}{2}(1+\theta)-d & \text { for } p \text { and } q \text { both odd. }\end{cases}
$$

Proof. By Theorem 2.1, we obtain

$F(q) \equiv \frac{1}{2}(q \theta-\alpha)=p / 2+d \equiv d \quad$ for $p$ and $q$ both even,

$F(q) \equiv \frac{1}{2}(\theta-q \theta+\alpha)=\theta / 2-p / 2-d \equiv \theta / 2-d \quad$ for $p$ even and $q$ odd,

$F(q) \equiv \frac{1}{2}(q \theta-\alpha)=p / 2+d \equiv 1 / 2+d \quad$ for $p$ odd and $q$ even,

$F(q) \equiv \frac{1}{2}(\theta-q \theta+\alpha)=\theta / 2-p / 2-d$

$\equiv \frac{1}{2}(1+\theta)-d \quad$ for $p$ and $q$ both odd.

Theorem 3.3. Let $p / q, q>1$ be a best $\alpha$-approximation to $\theta$.

(1) There exists exactly one vertex $v(q)$ with $\|q \theta-\alpha\|=2\|F(q)-v(q)\|$, and

$$
v(q)= \begin{cases}v_{0} & \text { for } p \text { and } q \text { both even, } \\ v_{1} & \text { for } p \text { even and } q \text { odd } \\ v_{2} & \text { for } p \text { odd and } q \text { even } \\ v_{3} & \text { for } p \text { and } q \text { both odd. }\end{cases}
$$

(2) If $F(1)=0$, then

$$
F(q)= \begin{cases}v_{1}-d & \text { for } p \text { even and } q \text { odd, } \\ v_{2}+d & \text { for } p \text { odd and } q \text { even, } \\ v_{3}-d & \text { for } p \text { and } q \text { both odd, }\end{cases}
$$

where $2 d=q \theta-p$.

Proof. Let $q>1$. Since $p / q$ is a best $\alpha$-approximation to $\theta, q$ is a critical index of the sequence $\|j \theta-\alpha\|$. By Theorem 3.2(1), $q$ is a critical index of the sequence $A(j), j \in \mathbb{N}$, and $\|q \theta-\alpha\|=2 A(q)$. Hence there exists a vertex $v(q) \in\left\{0, \frac{1}{2} \theta, \frac{1}{2}, \frac{1}{2}(1+\theta)\right\}$ such that $\|q \theta-\alpha\|=2\|F(q)-v(q)\|$. By Remark 3.1, $\|F(q)\|=\|F(q-1)\|$ and $\left\|F(q)-\frac{1}{2}\right\|=\left\|F(q-1)-\frac{1}{2}\right\|$ for $q$ odd, 
and $\left\|F(q)-\frac{\theta}{2}\right\|=\left\|F(q-1)-\frac{\theta}{2}\right\|$ and $\left\|F(q)-\frac{1}{2}(1+\theta)\right\|=\left\|F(q-1)-\frac{1}{2}(1+\theta)\right\|$ for $q$ even. Hence we have

$$
\begin{cases}v(q) \in\left\{\frac{\theta}{2}, \frac{1}{2}(1+\theta)\right\} & \text { for } q \text { odd } \\ v(q) \in\left\{0, \frac{1}{2}\right\} & \text { for } q \text { even. }\end{cases}
$$

Since $\left\|F(q)-\frac{\theta}{2}\right\|+\left\|F(q)-\frac{1}{2}(1+\theta)\right\|=\|F(q)\|+\left\|F(q)-\frac{1}{2}\right\|=\frac{1}{2}$ and $\|F(q)-v(q)\|=\frac{1}{2}\|q \theta-\alpha\|<\frac{1}{2}\|\theta-\alpha\| \leq \frac{1}{4}, v(q)$ is uniquely determined by (i). Thus by Lemma 3.4 we obtain condition (1).

If $F(1)=0$, then by $\mathrm{CF}(3)$ of Remark $3.3, p, q$ are relatively prime. Since $|q \theta-p|=\|q \theta\|<\|\theta\|$, we obtain $\frac{1}{2}(1+\theta+|q \theta-p|)<1$ and $\frac{1}{2}(\theta-|q \theta-p|)>0$. Hence by Lemma 3.4 we obtain condition (2).

ExAMPLE 3.1. Let $F(1)=0$ and $\theta=t / m$ be a fraction in lowest terms. Since $m$ is the last critical index of the sequence $\|j t / m\|, j \in \mathbb{N}$, Theorem 3.2(3) implies that $C(j)>0$ and $B(j)>0$ for $1 \leq j<m$. Hence by Theorem 2.1 we obtain the following conditions (1) and (2). Theorem 3.3(2) yields $(3)$.

(1) $\{F(1), F(2), \ldots, F(m)\}=\left\{0, \frac{1}{m}, \ldots, \frac{m-1}{m}\right\}$,

(2) $\min \left(\left\|F(j)-\frac{t}{2 m}\right\|,\left\|F(j)-\frac{1}{2}\right\|,\left\|F(j)-\frac{1}{2}\left(1+\frac{t}{m}\right)\right\|\right) \geq \frac{1}{2 m}, 1 \leq j<m$,

(3) $F(m)= \begin{cases}\frac{t}{2 m} & \text { for } t \text { even, } \\ \frac{1}{2} & \text { for } m \text { even, } \\ \frac{1}{2}\left(1+\frac{t}{m}\right) & \text { for } t \text { and } m \text { both odd. }\end{cases}$

By condition $\mathrm{CF}(1)$ one may state the results of Theorem 3.3 in terms of partial quotients.

Corollary 3.2. Let $F(1)=0$ and $\theta=\left[0 ; a_{1}, a_{2}, \ldots\right]<1 / 2$. If $q_{n}$ is the increasing sequence of all critical indices of the sequence $\|j \theta\|, j \in \mathbb{N}$, and $v\left(q_{n}\right), n \in \mathbb{N}$, is the sequence of vertices such that $\left\|q_{n} \theta\right\|=2\left\|F\left(q_{n}\right)-v\left(q_{n}\right)\right\|$, then

(1) $v\left(q_{1}\right)=\theta / 2, v\left(q_{2}\right)= \begin{cases}\frac{1}{2} & \text { for } a_{1} \text { even }, \\ \frac{1}{2}(1+\theta) & \text { for } a_{1} \text { odd }\end{cases}$

(2) $v\left(q_{n+2}\right)=v\left(q_{n}\right)$ iff $a_{n+1}$ is even,

(3) $v\left(q_{n+1}\right) \neq v\left(q_{n}\right)$.

Proof. Let $p_{n} / q_{n}$ be the $n$th convergent to $\theta$. By $\mathrm{CF}(1), p_{1}=0, p_{2}=1$ and $q_{2}=a_{1}$. Hence by Theorem 3.3 we obtain (1).

If $a_{n+1}$ is even, then by $\mathrm{CF}(1), p_{n+2} \equiv p_{n}$ and $q_{n+2} \equiv q_{n}$. Thus, by Theorem 3.3, $v\left(q_{n+2}\right)=v\left(q_{n}\right)$. If $a_{n+1}$ is odd, then $\mathrm{CF}(1)$ yields $p_{n+2} \equiv$ $p_{n+1}+p_{n}$ and $q_{n+2} \equiv q_{n+1}+q_{n}$. Since $p_{n+1}$ or $q_{n+1}$ is odd, we have $p_{n+2} \not \equiv p_{n}$ or $q_{n+2} \not \equiv q_{n}$. Thus, $v\left(q_{n+2}\right) \neq v\left(q_{n}\right)$ by Theorem 3.3. 
We prove (3) by induction. Notice that $v\left(q_{2}\right) \neq v\left(q_{1}\right)$. Assume that $v\left(q_{n+1}\right) \neq v\left(q_{n}\right)$. If $a_{n+1}$ is even, then $v\left(q_{n+2}\right)=v\left(q_{n}\right) \neq v\left(q_{n+1}\right)$ by (2). If $a_{n+1}$ is odd, then by $\mathrm{CF}(1)$ we have $v\left(q_{n+2}\right) \neq v\left(q_{n+1}\right)$ analogously as in $(2)$.

Corollary 3.3. Let $F(1)=0$ and let $\theta<1 / 2$ be irrational. If $q_{n}$ is an increasing sequence of critical indices of the sequence $\|j \theta\|, j \in \mathbb{N}$, and $v\left(q_{n}\right)$ is a sequence of vertices such that $\left\|q_{n} \theta\right\|=2\left\|F\left(q_{n}\right)-v\left(q_{n}\right)\right\|$, then

$$
\begin{aligned}
& \operatorname{sgn}\left(F\left(q_{n+1}\right)-v\left(q_{n+1}\right)\right)=\operatorname{sgn}\left(F\left(q_{n}\right)-v\left(q_{n}\right)\right) \quad \text { iff } \\
& v\left(q_{n+1}\right)=1 / 2 \text { or } v\left(q_{n}\right)=1 / 2 .
\end{aligned}
$$

Proof. By Corollary 3.2(3), $v\left(q_{n+1}\right) \neq v\left(q_{n}\right)$. Hence by Theorem 3.3(2) and condition $\mathrm{CF}(2)$ we obtain $(*)$.

This corollary shows that a billiard ball, starting from vertex 0 , changes the orientation of its trajectory at the points $F\left(q_{n}\right)$ by the rule $(*)$.

ExAmple 3.2. Suppose $F(1)=0, \theta=\left[0 ; a_{1}, a_{2}, \ldots\right]<1 / 2, a_{1}$ is even and $a_{n}$ is odd for $n \geq 2$. If $q_{n}, n \in \mathbb{N}$, is the increasing sequence of all critical indices of the sequence $\|j \theta\|, j \in \mathbb{N}$, then by Corollary 3.2 the sequence $v\left(q_{n}\right)$, $n \in \mathbb{N}$, is 3 -periodic:

$$
v\left(q_{1}\right)=\theta / 2, \quad v\left(q_{2}\right)=1 / 2, \quad v\left(q_{3}\right)=\frac{1}{2}(1+\theta),
$$

and by Corollary 3.3 the sequence $\operatorname{sgn}\left(F\left(q_{n}\right)-v\left(q_{n}\right)\right), n \in \mathbb{N}$, is 6-periodic:

$$
\begin{aligned}
& \operatorname{sgn}\left(F\left(q_{1}\right)-v\left(q_{1}\right)\right)=\operatorname{sgn}\left(F\left(q_{2}\right)-v\left(q_{2}\right)\right)=\operatorname{sgn}\left(F\left(q_{3}\right)-v\left(q_{3}\right)\right)=-1, \\
& \operatorname{sgn}\left(F\left(q_{4}\right)-v\left(q_{4}\right)\right)=\operatorname{sgn}\left(F\left(q_{5}\right)-v\left(q_{5}\right)\right)=\operatorname{sgn}\left(F\left(q_{6}\right)-v\left(q_{6}\right)\right)=1 .
\end{aligned}
$$

\section{References}

[1] P. Alessandri and V. Berthe, Three distance theorem and combinatorics on words, Enseign. Math. 44 (1988), 103-132.

[2] E. S. Barnes and H. P. F. Swinnerton-Dyer, The inhomogeneous minima of binary quadratic forms, I-III, Acta Math. 87 (1952), 259-323; 88 (1952), 279-316; 92 (1954), 199-234.

[3] J. W. S. Cassels, Über $\varliminf_{x \rightarrow+\infty} x|\vartheta x+\alpha-y|$, Math. Ann. 127 (1954), 288-304.

[4] -, An Introduction to Diophantine Approximation, Cambridge Univ. Press, 1957.

[5] T. W. Cusick, A. M. Rockett and P. Szüsz, On inhomogeneous Diophantine approximation, J. Number Theory 48 (1994), 259-283.

[6] J. F. Geelen and R. J. Simpson, A two dimensional Steinhaus theorem, Australas. J. Combin. 8 (1993), 169-197.

[7] A. Ya. Khintchine, Über eine Klasse linearer diophantischer Approximationen, Rend. Circ. Mat. Palermo 50 (1926), 170-195.

[8] T. Komatsu, On inhomogeneous diophantine approximation and the Nishioka-Shiokawa-Tamura algorithm, Acta Arith. 86 (1998), 305-324.

[9] J. L. Lagrange, Traité de la résolution des équations numériques de tous les degrés, 1st ed., Paris, 1798; 2nd ed., 1808; reprinted 1826, Chapter VI, §68. 
[10] T. van Ravenstein, The three gap theorem (Steinhaus conjecture), J. Austral. Math. Soc. Ser. A 45 (1988), 360-370.

[11] V. T. Sós, On the theory of Diophantine approximations I, II, Acta Math. Acad. Sci. Hungar. 8 (1957), 461-472; 9 (1958), 229-241.

[12] -, On the distribution mod 1 of the sequence na, Ann. Univ. Sci. Budapest. Eötvös Sect. Math. 1 (1958), 127-134.

[13] J. Surányi, Über die Anordnung der Vielfachen einer reellen Zahl mod 1, ibid., 107-111.

[14] S. Świerczkowski, On successive settings of an arc on the circumference of a circle, Fund. Math. 46 (1958), 187-189.

Institute of Mathematics

University of Economics

Komandorska 118/120

53-345 Wrocław, Poland

E-mail: jan.florek@ae.wroc.pl

Received on 8.6.2007

and in revised form on 30.4.2008 